\title{
Extended DLVO theory: Electrostatic and non-electrostatic forces in oxide suspensions
}

\author{
M. Boström ${ }^{\mathrm{a}, *}$, V. Deniz ${ }^{\mathrm{a}}$, G.V. Franks ${ }^{\mathrm{b}}$, B.W. Ninham ${ }^{\mathrm{c}}$ \\ ${ }^{a}$ Department of Physics and Measurement Technology, Linköping University, SE-581 83 Linköping, Sweden \\ ${ }^{\mathrm{b}}$ Chemical and Biomolecular Engineering, University of Melbourne, 3010 Vic, Australia \\ ${ }^{\mathrm{c}}$ Research School of Physical Sciences and Engineering, Australian National University, Canberra, ACT 0200, Australia
}

Available online 27 June 2006

It is a pleasure to dedicate this work to Dr. Kash Mittal on his 60th birthday.

\begin{abstract}
According to classical DLVO theory all ions of background salt solution with the same ionic charge should result in the same effective force between colloidal particles. However, the relative effectiveness of different ions in influencing forces between ceramic oxide surfaces follows either a reversed Hofmeister sequence or a direct Hofmeister sequence depending on the type of oxide and if the pH is above or below the isoelectric point (iep). This ion specificity is inexplicable in classical double layer theory that deals only with pure electrostatic forces acting between the ions and the colloidal particles. A theoretical explanation is given here. At, and above, biological salt concentrations other, nonelectrostatic (NES) ion specific forces act that are ignored in such modeling. In this overview we present the basic theory for the double layer near a single oxide surface and for the extended DLVO forces between oxide colloidal particles that accounts for these NES forces. We will demonstrate that ion specificity can be understood to a large degree once NES forces are included consistently in the non-linear theory.

(C) 2006 Elsevier B.V. All rights reserved.
\end{abstract}

\section{Contents}

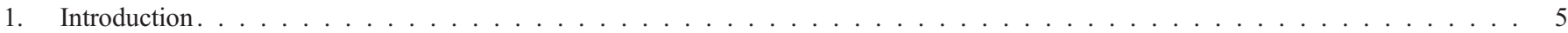

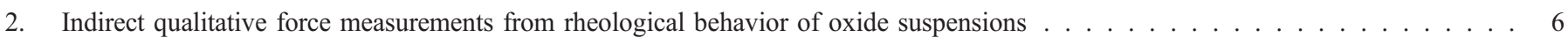

3. Non-electrostatic forces acting on ions near a charged interface . . . . . . . . . . . . . . . . . . . . . . . 7

4. The extended double layer theory near a single interface . . . . . . . . . . . . . . . . . . . . . . . 8

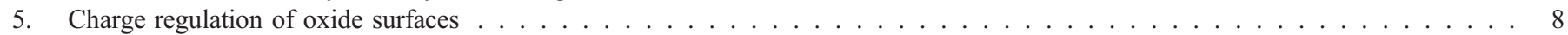

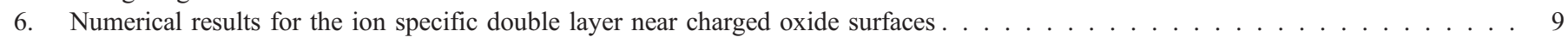

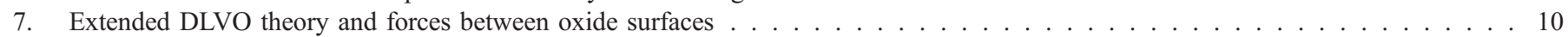

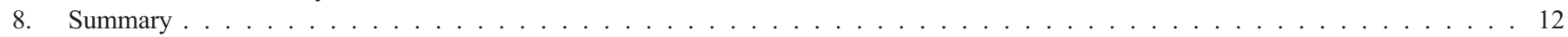

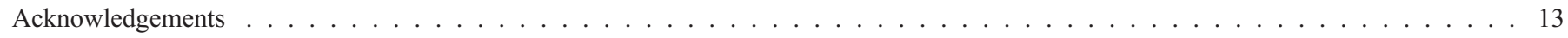

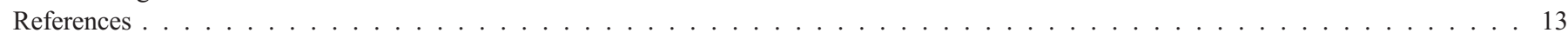

\section{Introduction}

To understand colloid science, the behavior of surfactants in solution, molecular biology and industrial processes that takes place in solution it is essential to understand interactions bet-

* Corresponding author. Fax: +4613137568.

E-mail address: mabos@ifm.liu.se (M. Boström). ween particles in solution. However, as it turns out an understanding of the very basic underlying foundation in colloid science has been missing. The pioneering work of Hofmeister [1] showed that crystallization of proteins depended not only on charge but also on the specific choice of salt solution. This is true even when salt ions have the same ionic charge. These effects have, for instance, been observed in experiments as diverse as double layer force measurements [2-10], bubble fusion [11], 
conformational changes of rhodopsin [12], bacterial cell growth [13], yield stresses in silica and alumina suspensions [14-17], cutting-efficiency of DNA by restriction enzymes [18], charge of globular proteins [19], surface tension of electrolytes [20-23] and the solubility of protein solutions [24-27]. The relative effectiveness of different anions in crystallizing proteins follows a reversed Hofmeister sequence for $\mathrm{pH}$ below the isoelectric point (pI), and a direct Hofmeister sequence for $\mathrm{pH}>\mathrm{pI}$ [24-27]. Such phenomenon has been known almost since Hofmeister's original work but it has not been understood. The only ionic characteristics included in textbook descriptions of colloids and salt solutions are bulk $\mathrm{pH}$, salt concentration, ionic charges and "hydrated" ionic radii. Electrostatic theories based on these parameters do not account for the experimentally observed ion specificity. Since standard theory failed, it has been customary to invoke various specific "ionic characteristics" such as "structure breaking", "structure creating", "lyotropic", "kosmotropic", "chaotropic", "salting-in", and "salting-out" [28,29]. These concepts attempt to, and do encompass the missing ion-solvent interactions qualitatively. Other authors suggest gel-like layers [30-32], or "fragile" structure [33], as soft surface layers to account for short range repulsion, particularly for silica. But all these concepts have turned out to be difficult to quantify.

Ion specificity means that it is possible to manipulate forces between colloidal particles not only by changing the $\mathrm{pH}$ (surface charge) and salt concentration (screening), but also by changing ionic species. However, the reason until very recently was not understood. The ion specific effects reveal a systematic behavior that suggests that predictive theories should be within reach. This may, in fact, very well be so. There are at, and above, biological salt concentrations other, non-electrostatic (NES) ion specific forces acting that are ignored in such modeling. These missing forces are variously discussed under familiar, but generally unquantified, terms, such as, hydration, hydrogen bonding, $\pi$-electron-cation interactions, dipole-dipole, dipole-induced dipole and induced dipole-induced dipole forces and so on. The important contribution from many-body electrodynamic fluctuation force is accessible from extensions of the Lifshitz theory from which, with relevant dielectric susceptibility data on solutions as a function of frequency, the forces can be extracted quantitatively. We will describe how some of the other unquantified forces also can be estimated from the extended Lifshitz theory due to Mahanty and Ninham [34] that takes ion size, finite temperature, and the presence of screening charges into account. The classical theories of colloid science that ignore such contributions do not account for ion specific phenomena. The purpose of this paper is to present results that may explain some of these effects in oxide particle suspensions. We present calculations for the force between oxide surfaces using a modified Poisson-Boltzmann equation that goes beyond electrostatics in that it includes also non-electrostatic ion-colloid dispersion potentials. These dispersion potentials originate from polarizabilities of both ions and colloids. In the past these dispersion potentials have usually been neglected but it is now established that they are vital, especially at typical biological salt concentrations $(0.1 \mathrm{M}$ and above) where electrostatics is screened [1,3537]. We will show here how some of the highly ion specific experimental results obtained by Franks et al. can be understood [14-17].

The outline of this paper is as follows. We briefly describe what we learn about ion specificity from rheological and other experiments with oxide suspension in Section 2. We then describe the non-electrostatic forces that act on ions near a single charged planar surface in Section 3. The extended double layer theory near a single interface is presented in Section 4 and we summarize briefly in Section 5 the theory of charge regulation [38] of oxide surfaces in electrolyte solutions. In Section 6 we present numerical results for ion distributions near oxide surfaces for various salt solutions and $\mathrm{pH}$. Then in Section 7 we present the basic theory of ion specific extended DLVO theory and present numerical results for forces between two oxide surfaces $\left(\mathrm{SiO}_{2}\right.$ and $\left.\alpha-\mathrm{Al}_{2} \mathrm{O}_{3}\right)$ under different conditions. Finally, in Section 8, we summarize our results and the derivation of the expression for the extended DLVO pressure is presented in Appendix A.

\section{Indirect qualitative force measurements from rheological behavior of oxide suspensions}

Rheological measurements on sub-micrometer suspensions can be used to qualitatively determine, to some extent, the forces acting between particles in a particular solution. It is well known that suspension viscosity is low and nearly Newtonian for well dispersed suspensions (net inter-particle repulsion) at moderate volume fractions (15-40 vol.\% solids) [39]. When the net interparticle interaction is attractive the shear thinning viscosity of suspension increased and has solid-like characteristics such as elasticity and yield stress. Both yield stress and elastic modulus increase as the magnitude of attraction increases [39]. Thus by measuring the yield stress of a suspension one can determine if the net force between particles is repulsive or attractive and can determine the relative magnitude of attraction when all other factors are maintained constant.

Franks et al. have found that the yield stress of oxide suspensions usually follows a direct or reversed Hofmeister sequence. For low isoelectric point materials such as silica, at $\mathrm{pH}$ above the iep, the yield stress increases according to a sequence of monovalent cations $\mathrm{Li}<\mathrm{Na}<\mathrm{K}<\mathrm{Cs}$ [14] when salt concentration is increased above about $0.1 \mathrm{M}$. This normal Hofmeister sequence is consistent with the preferred binding of cations according to the following sequence $\mathrm{Cs}>\mathrm{K}>\mathrm{Na}>\mathrm{Li}$ [40]. These rheological results suggest that the magnitude of attraction between silica surfaces (in high concentration of a monovalent electrolyte) increases according to the following $\mathrm{Cs}>\mathrm{K}>\mathrm{Na}>\mathrm{Li}$. On the other hand, high iep, materials such as alumina, show reverse Hofmeister sequences [41]. At pH above alumina's iep, the yield stresses and (presumed) magnitude of attraction decreases according to $\mathrm{Li}>\mathrm{Na}>\mathrm{K}>\mathrm{Cs}$ [15]. At $\mathrm{pH}$ below their iep, alumina and zirconia, have yield stresses that decrease according to the following sequence of monovalent anions $\mathrm{IO}_{3}>\mathrm{BrO}_{3}>$ $\mathrm{NO}_{3} \approx \mathrm{Cl}>\mathrm{ClO}_{4}[16]$.

Based on the rheological evidence presented, one would expect the net inter-particle potential energy between two silica surfaces and between two alumina surfaces at between about 0.2 
and $1.0 \mathrm{M}$ monovalent salts to qualitatively have the trends shown in Fig. 1. One of the most comprehensive of the few reports on direct measurement of surface forces [8] in concentrated $(>0.2 \mathrm{M})$ electrolyte for silica is limited to $\mathrm{pH} 5.6$ and only one surface is silica, the other is mica. At $\mathrm{pH} 5.6$ where the forces were measured, the rheological results suggest that the Hofmeister sequence is reversed from that shown in Fig. 1, so that the force measurements [8] are in agreement with the rheological behaviour [14] but the sequence depends on the $\mathrm{pH}$.

\section{Non-electrostatic forces acting on ions near a charged interface}

It has been customary to assume that only electrostatic forces have an effect on ions in solution. However, these forces are the same for all ions with the same ionic charge and do not explain the experimentally observed ion specificity. Ninham and Yaminsky [35] showed that there were other non-electrostatic forces of electrodynamic origin that successfully competed with the screened electrostatic forces at and above biological salt concentrations. We will show here how these non-electrostatic forces can be quantified. Mahanty and Ninham demonstrated [34] how the forces acting on finite size polarizable particles due to electrodynamic fluctuations could be described. The dispersion self-energy of a polarizable particle (e.g. an ion) of finite size a as a function of the distance $x$ from the interface can be written as a sum of the following two terms [34],

$$
\begin{aligned}
& U_{0}(x)=\frac{2 \hbar}{\pi^{3 / 2} a^{3}} \int_{0}^{\infty} \mathrm{d} \xi \alpha^{*}(i \xi)\left\{\frac{\theta(x)}{\varepsilon_{2}(i \xi)}+\frac{\theta(-x)}{\varepsilon_{1}(i \xi)}\right\}, \\
& U_{1}(x)=\frac{\hbar}{\pi^{3 / 2} a^{3}} \int_{0}^{\infty} \mathrm{d} \xi \alpha^{*}(i \xi) \Delta_{12}(i \xi)\left\{\frac{\theta(-x)}{\varepsilon_{1}(i \xi)}-\frac{\theta(x)}{\varepsilon_{2}(i \xi)}\right\} \times f(x),
\end{aligned}
$$

where

$$
\begin{aligned}
& f(x)=e^{-x^{2} / a^{2}}-\frac{\sqrt{\pi}|x|}{a} \operatorname{erfc}\left(\frac{|x|}{a}\right)+\frac{a^{3}}{2|x|^{3}} \int_{0}^{|x| / a}\left(e^{-t^{2}}-e^{-x^{2} / a^{2}}\right), \\
& \Delta_{12}(i \xi)=\frac{\varepsilon_{1}(i \xi)-\varepsilon_{2}(i \xi)}{\varepsilon_{1}(i \xi)+\varepsilon_{2}(i \xi)} .
\end{aligned}
$$

The first energy term is the dispersion self-energy infinitely far from the interface and the second term is the modification to this self-energy due to the presence of the interface. Here the dielectric functions of water $\left(\varepsilon_{2}(i \xi)\right)$ and oxide surface $\left(\varepsilon_{1}(i \xi)\right)$, as well as the excess polarizability of the polarizable ion $\left(\alpha^{*}(i \xi)\right)$, all depend on frequency. The excess polarizability of an ion is the difference between the polarizability of the ion and the surrounding water. $\theta(z)$ is the step function which is unity when the argument is positive and zero when it is negative. The excess polarizabilities were taken from Tavares et al. [37]. The model dielectric functions of water [42], $\mathrm{SiO}_{2}[43]$ and $\alpha-\mathrm{Al}_{2} \mathrm{O}_{3}$ [44] were taken from the literature. The magnitudes, and even the signs, of the dispersion potentials near an interface depend in a sensitive way on these frequency-dependent quantities.

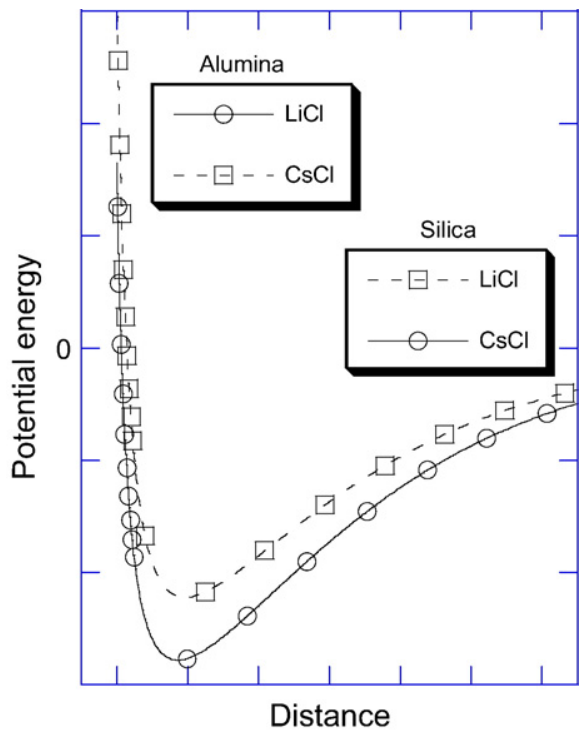

Fig. 1. Schematic illustration of the interparticle potential energy for two alumina or silica surfaces in approximately $0.3-1.3 \mathrm{M}$ monovalent chlorides based on the rheological evidence. Note that the two curves have different meaning for the different oxides. For alumina the schematic is developed for $\mathrm{pH}$ about 12 and for silica the schematic is for $\mathrm{pH}$ about 8 . At other $\mathrm{pH}$ values the sequence may be different.

At finite temperatures the normal electrodynamic modes that give rise to the dispersion energy are thermally populated [4549]. The finite temperature free energy can be obtained from the corresponding zero temperature energy with the following substitution:

$$
\begin{aligned}
& \frac{\hbar}{2 \pi} \int_{0}^{\infty} f(i \xi) \mathrm{d} \xi \rightarrow k_{\mathrm{B}} T \sum_{\xi_{n}} f\left(i \xi_{n}\right), \quad \xi_{m}=2 \pi k_{\mathrm{B}} T n / \hbar, \\
& \quad n=0,1,2, \ldots,
\end{aligned}
$$

i.e. one obtains the result as a summation along the imaginary frequency axis and the prime indicates that the $n=0$ term should be reduced by a factor of two. We will in the following sections approximate the dispersion potential with the result far from the interface, which is

$U_{\text {dispersion }}(x)=B / x^{3}$

$B \approx \frac{-k_{\mathrm{B}} T}{2} \sum_{n=0}^{\infty} \frac{\alpha^{*}(i \xi) \Delta_{12}(i \xi)}{\varepsilon_{2}(i \xi)}$.

The estimated $B$-values for the considered ions and surface combinations are given in Table 1. Mahanty and Ninham [34] showed that the $n=0$ term in the frequency summation becomes screened in the presence of charged electrolyte ions. The higher frequency terms $(n>0)$, on the other hand, are unaffected by the presence of electrolyte since they are evaluated at frequencies considerably higher than the ion fluctuations.

Parsegian and Ninham showed that the finite temperature extension of the Lifshitz formalism could accommodate several other previously more or less unquantified forces [45]. One 
Table 1

The estimated $B$-values used to calculate the ionic dispersion potential for selected ion-surface combinations

\begin{tabular}{lll}
\hline Ion & $\mathrm{SiO}_{2}$ & $\mathrm{Al}_{2} \mathrm{O}_{3}$ \\
\hline $\mathrm{Li}^{+}$ & $-1.0 \times 10^{-51} \mathrm{~J} \mathrm{~m}^{3}$ & $-2.0 \times 10^{-51} \mathrm{~J} \mathrm{~m}^{3}$ \\
$\mathrm{Na}^{+}$ & $-4.1 \times 10^{-51} \mathrm{~J} \mathrm{~m}^{3}$ & $-8.5 \times 10^{-51} \mathrm{~J} \mathrm{~m}^{3}$ \\
$\mathrm{~K}^{+}$ & $-1.6 \times 10^{-50} \mathrm{~J} \mathrm{~m}^{3}$ & $-3.5 \times 10^{-50} \mathrm{~J} \mathrm{~m}^{3}$ \\
$\mathrm{Cs}^{+}$ & $-3.3 \times 10^{-50} \mathrm{~J} \mathrm{~m}^{3}$ & $-7.6 \times 10^{-50} \mathrm{~J} \mathrm{~m}^{3}$ \\
$\mathrm{Cl}^{-}$ & $-2.4 \times 10^{-50} \mathrm{~J} \mathrm{~m}^{3}$ & $-6.2 \times 10^{-50} \mathrm{~J} \mathrm{~m}^{3}$ \\
$\mathrm{Br}^{-}$ & $-2.9 \times 10^{-50} \mathrm{~J} \mathrm{~m}^{3}$ & $-7.8 \times 10^{-50} \mathrm{~J} \mathrm{~m}^{3}$ \\
$\mathrm{I}^{-}$ & $-3.6 \times 10^{-50} \mathrm{~J} \mathrm{~m}^{3}$ & $-9.9 \times 10^{-50} \mathrm{~J} \mathrm{~m}^{3}$ \\
\hline
\end{tabular}

example is proton fluctuations in the microwave region that can produce an effective attraction between proteins [45]. These Kirkwood-Shumaker forces are included in the finite temperature Lifshitz theory through the frequency dependence of the dielectric function of a dilute protein solution [45]. In the low density limit the Keesom forces acting between permanent dipolar molecules and the Debye forces due to dipole-induced dipole interactions are also included in the same Lifshitz expression [34]. So we emphasize that with the term "dispersion forces" we refer in general not only to high frequency (optical and UV) van der Waals dispersion forces but also KirkwoodShumaker, Keesom, Debye and other contributions. All these contributions can be accessed through the same general formalism [34]. The ion solvation energy is the change in selfenergy in bringing an ion from one medium to another. We have recently explored how dispersion self free energies contribute to the solvation energy of ions in solution [50], to ion transfer across low dielectric membranes [51], and to solvation energy changes as ions move into regions with varying water density [22]. The last is particularly interesting for our discussion of non-electrostatic forces acting on ions near interfaces. We obtained an approximate expression for the change in solvation energy $\left(U_{\text {solvation }}\right)$ in bringing an ion from water to air through the surface region,

$U_{\text {solvation }}(x) \approx \Delta G_{\text {solvation }} \frac{\frac{1}{1+p(x)\left[\varepsilon_{2}(0)-1\right]}-\frac{1}{\varepsilon_{2}(0)}}{1-\frac{1}{\varepsilon_{2}(0)}}$

Here $p(x)$ is the water density profile that can be obtained from simulations or from experiments. This means that changes in dielectric properties of the background media near an interface can also be taken into account in our extended DLVO theory. One can in the same way include effects of a hydrated ion size that depends on the distance to the surface. The classical theories of colloid science which miss such contributions do not account for a whole variety of ion specific phenomena. In particular, for example, the interfacial tension of salt water as a function of concentration and salt type $[21,22]$ and the specificity of ion transport across low dielectric media [51] are not accounted for by electrostatic theories alone. The inclusion of these NES specific ion forces requires that they be treated on the same level and in the same framework as electrostatic forces in the nonlinear double layer theory. We carry out such program in the following sections.

\section{The extended double layer theory near a single interface}

At chemical equilibrium the ions in solution are distributed according to a Boltzmann relation [35],

$c_{i}(x)=c_{i, 0} \exp \left[-\left(z_{i} e \phi(x)+U_{i}(x)\right)\right]$,

where $c_{i, 0}$ and $z_{i}$ denote, respectively, the bulk concentration and valency of ionic species $i$. Besides the electrostatic self-consistent potential $(\phi(x))$ each ion is also acted upon by image potentials and various non-electrostatic forces $\left(U_{i}(x)\right)$. At and above biological concentrations the image potential is smaller than the ionic dispersion potential at all ion-surface distances [52]. We approximate in this work $U_{i}(x)$ with the ionic dispersion potential given in Eq. (6). The self-consistent electrostatic potential can be found from the Poisson equation, as:

$\frac{\mathrm{d}^{2} \phi}{\mathrm{d} x^{2}}=-\frac{\rho(x)}{\varepsilon_{0} \varepsilon_{2}(0)}$,

$\rho(x)=e \sum_{i} z_{i} c_{i}(x)$

When combined together, Eqs. (9)-(11) give the modified Poisson-Boltzmann equation. This equation can be solved numerically after one has decided on the appropriate boundary conditions. In the case of a single oxide surface the electrostatic field should go to zero at infinite separation from the surface. For the case of two equal interacting oxide surfaces considered later the electric field goes to zero in the midplane between the plates. There are three commonly assumed boundary conditions at the surface: (i) constant surface potential, (ii) constant surface charge, and (iii) charge regulated surface. It is the third boundary condition (first described by Ninham and Parsegian [38]) that is relevant for oxide surfaces. This charge regulation boundary condition will be described in the next section.

\section{Charge regulation of oxide surfaces}

Surface charge is an important quantity that influences the stability and rheology of dispersions of ceramic oxide particles in aqueous electrolyte solutions. Oxide colloidal particles in solution can become charged by adsorption or desorption of potential determining ions. We will consider two examples, alumina oxide and silica oxide surfaces. Reactions that can be described with the Ninham-Parsegian charge regulation model [38] occur at surface hydroxyl groups (which are created in reactions with water molecules: $\left.\mathrm{Al}+\mathrm{AlO}+\mathrm{H}_{2} \mathrm{O} \leftrightarrow 2 \mathrm{AlOH}\right)$. For an $\alpha-\mathrm{Al}_{2} \mathrm{O}_{3}$ surface the interactions can be described as follows [53],

$$
\mathrm{AlOH}+\mathrm{H}_{\mathrm{s}}^{+} \leftrightarrow \mathrm{AlOH}_{2}^{+}\left(K_{\mathrm{a} 1}=\frac{[\mathrm{AlOH}]}{\left[\mathrm{AlOH}_{2}^{+}\right]}\left[\mathrm{H}_{\mathrm{s}}^{+}\right]=10^{-\mathrm{p} K_{\mathrm{al}}}\right)
$$

$$
\mathrm{AlOH} \leftrightarrow \mathrm{AlO}^{-}+\mathrm{H}_{\mathrm{s}}^{+}\left(K_{\mathrm{a} 2}=\frac{\left[\mathrm{AlO}^{-}\right]}{[\mathrm{AlOH}]}\left[\mathrm{H}_{\mathrm{s}}^{+}\right]=10^{-\mathrm{p} K_{\mathrm{a} 2}}\right)
$$


It is the proton concentration near the interface that is important,

$\left\lfloor\mathrm{H}_{\mathrm{s}}^{+}\right\rfloor=\left\lfloor\mathrm{H}^{+}\right\rfloor \exp \left\{-\left[e \phi(\right.\right.$ surface $)+U_{\mathrm{H}}($ surface $\left.\left.)\right]\right\}$,

$\left\lfloor\mathrm{H}^{+}\right\rfloor=10^{-\mathrm{pH}} / \gamma_{\mathrm{H}} \approx 10^{-\mathrm{pH}}$,

where the last approximation is that the activity coefficient $\left(\gamma_{\mathrm{H}}\right)$ is close to unity. (At sufficiently low salt concentration the activity coefficient is approximately given by $\left.\gamma_{\mathrm{H}} \approx \exp \left[-\left(\frac{e^{2}}{8 \pi \varepsilon_{0} \varepsilon_{2}(0) k T}\right) \frac{\kappa}{1+2 a \kappa}+\frac{8 \pi}{3} c(2 a)^{3}\right]\right)$. The surface electrostatic potential that acts on hydronium ions in close contact with the interface will be ion specific as discussed in the previous section. There are, in general, also non-electrostatic forces acting on the hydronium ions which should be included in the estimation of the surface hydronium concentration. We have here neglected this contribution (which corresponds to the same constant shift in bulk hydronium concentration for all considered examples). The surface charge of the alumina oxide surface can be written as,

$\sigma_{0}=e N_{\mathrm{s}} \frac{\left[\mathrm{AlOH}_{2}^{+}\right]-\left[\mathrm{AlO}^{-}\right]}{[\mathrm{AlOH}]+\left[\mathrm{AlOH}_{2}^{+}\right]+\left[\mathrm{AlO}^{-}\right]}$,

where $N_{\mathrm{s}}$ is the total site density. This can be rewritten in terms of the $\mathrm{p} K_{\mathrm{a}}$ values of the acidic and basic reactions using Eqs. (12) and (13),

$\sigma_{0}=e N_{\mathrm{s}} \frac{\left[\mathrm{H}_{\mathrm{s}}^{+}\right] 10^{\mathrm{p} K_{\mathrm{a} 1}}-\left[\mathrm{H}_{\mathrm{s}}^{+}\right]^{-1} 10^{-\mathrm{p} K_{\mathrm{a} 2}}}{1+\left[\mathrm{H}_{\mathrm{s}}^{+}\right] 10^{\mathrm{p} K_{\mathrm{a} 1}}+\left[\mathrm{H}_{\mathrm{s}}^{+}\right]^{-1} 10^{-\mathrm{p} K_{\mathrm{a} 2}}}$.

(In cases where the $\mathrm{p} K_{\mathrm{a}}$ values are far enough separated this expression can be approximated with a sum of two independent terms, one for the basic reaction and one for the acidic reaction). The same expressions hold for the $\mathrm{SiO}_{2}$ surface charge density. James [53] presented the values $\mathrm{p} K_{\mathrm{a} 1}=8.50, \mathrm{p} K_{\mathrm{a} 2}=9.70$, and $N_{\mathrm{s}}=2.7 \mathrm{sites} / \mathrm{nm}^{2}$ for an $\alpha-\mathrm{Al}_{2} \mathrm{O}_{3}$. This leads to an isoelectric point at $\mathrm{pH}$ 9.1. Similarly for $\mathrm{SiO}_{2}$ James [53] gave the values $\mathrm{p} K_{\mathrm{a} 1}=1.34, \mathrm{p} K_{\mathrm{a} 2}=4.6, N_{\mathrm{s}}=3.5$ sites $/ \mathrm{nm}^{2}$ and an isoelectric point at $\mathrm{pH} 3$. These $\mathrm{p} K_{\mathrm{a}}$ values are typically obtained as theoretical fits to experiments. Since the theory used to obtain these $\mathrm{p} K_{\mathrm{a}}$ values is incorrect we can only use them as estimates of the correct values to exemplify how inclusion of NES forces influences the result. The boundary condition for the PoissonBoltzmann equation is found by combining Eq. (16) with the following expression,

$\left.\frac{\mathrm{d} \phi}{\mathrm{d} x}\right|_{x=a}=\frac{-\sigma_{0}}{\varepsilon_{0} \varepsilon_{2}(0)}$.

Here $a$ is the ionic radius which we, for demonstrational purposes, take to be the same for all ions ( $2 \AA$ ). It is well known that the surface potential, and hence the surface charge, in the Ninham-Parsegian charge regulation model [38] depends on $\mathrm{pH}$ and the distance between two oxide surfaces. What has not been explored in the past is how the previously neglected non-electrostatic forces influence the surface charge and interaction between two oxide surfaces. This will now for the first time be explored in the following two sections.

\section{Numerical results for the ion specific double layer near charged oxide surfaces}

It has previously been shown that NES forces play a crucial role in the ion specificity observed in membrane biology [5456], ion binding to micelles [57] and polyelectrolytes [58], in water absorbency of wool fibers [59], and growth rates of Staphylococcus aureus and Pseudomonas aeruginosa [60]. We will here briefly present some examples that demonstrate the important influence of these NES forces on ion distributions near charged oxide surfaces.

We show in Fig. 2 the normalized chloride (co-ion) distributions near an alumina oxide surface at $\mathrm{pH} 10$ in different $0.4 \mathrm{M}$ salt solutions $(\mathrm{LiCl}, \mathrm{NaCl}, \mathrm{KCl}$, and $\mathrm{CsCl})$. The corresponding normalized counterion distributions are shown in Fig. 3. It is evident that the different ionic dispersion potentials acting on the counterions $\left(\mathrm{Li}^{+}, \mathrm{Na}^{+}, \mathrm{K}^{+}\right.$, and $\left.\mathrm{Cs}^{+}\right)$influence both the counterions and the co-ions. The influence on the coions is due to the non-linear coupling of the ions through the self-consistent electrostatic potential (which is influenced by the NES forces). In the same way it is possible to show that the coion distribution depends sensitively on the NES forces acting on different co-ions. Large attractive NES forces acting between counterions and the alumina oxide surfaces lead to strong counterion adsorption at the surface and a depletion of counterions in the middle of the two surfaces. This leads to an enhanced co-ion concentration between the surfaces, and as we will show in the next section it also leads to ion specific double layer pressures.

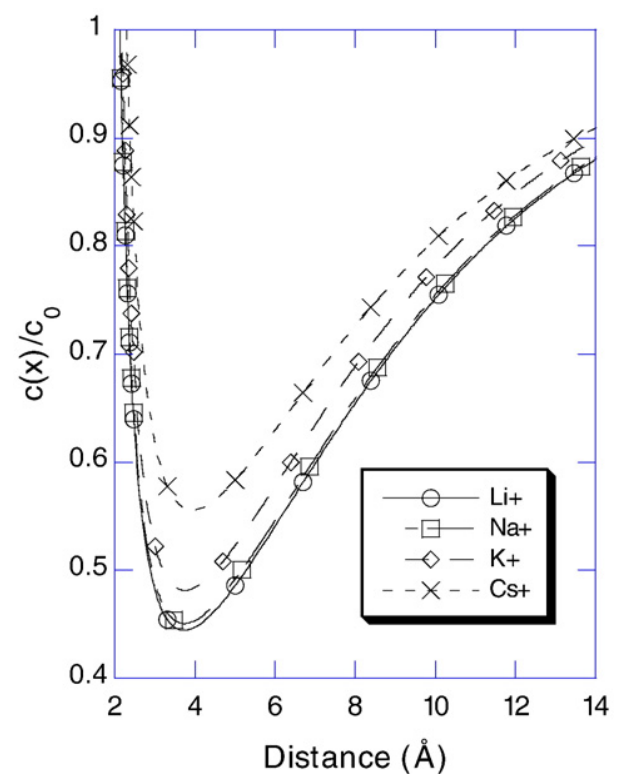

Fig. 2. Normalized $\mathrm{Cl}^{-}$co-ion distributions in different $0.4 \mathrm{M}$ salt solutions at $\mathrm{pH} 10$ near an $\mathrm{Al}_{2} \mathrm{O}_{3}$ surface. The different counterions used were: $\mathrm{Li}^{+}$(circles), $\mathrm{Na}^{+}$(squares), $\mathrm{K}^{+}$(diamonds), and $\mathrm{Cs}^{+}$(crosses). 


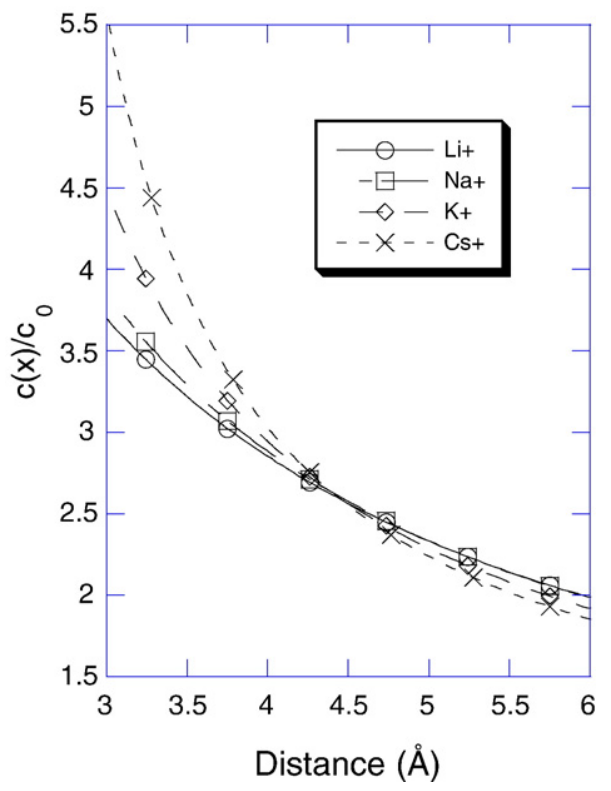

Fig. 3. Normalized counterion distributions in different $0.4 \mathrm{M}$ chloride salt solutions at $\mathrm{pH} 10$ near an $\mathrm{Al}_{2} \mathrm{O}_{3}$ surface. The different counterions used were: $\mathrm{Li}^{+}$(circles), $\mathrm{Na}^{+}$(squares), $\mathrm{K}^{+}$(diamonds), and $\mathrm{Cs}^{+}$(crosses).

One can see in Fig. 4 how the ion specific double layers also influence the surface charge density of two alumina oxide surfaces $(20 \AA$ apart) in $0.4 \mathrm{M} \mathrm{NaCl}, \mathrm{NaBr}$ and $\mathrm{NaI}$. More polarizable anions are more attracted towards the alumina surface which by electrostatics leads to an enhanced concentration of hydronium ions near the surface. In the $\mathrm{pH}$ range between 5 and 9 one can see that the surface charge density in different $0.4 \mathrm{M}$ salt solutions becomes more positive as we go from $\mathrm{NaCl}$ to $\mathrm{NaBr}$ and finally to $\mathrm{NaI}$. This increase of the surface charge density below the isoelectric point would suggest that the pressure should increase in the order $\mathrm{NaCl}<\mathrm{NaBr}<\mathrm{NaI}$. How-

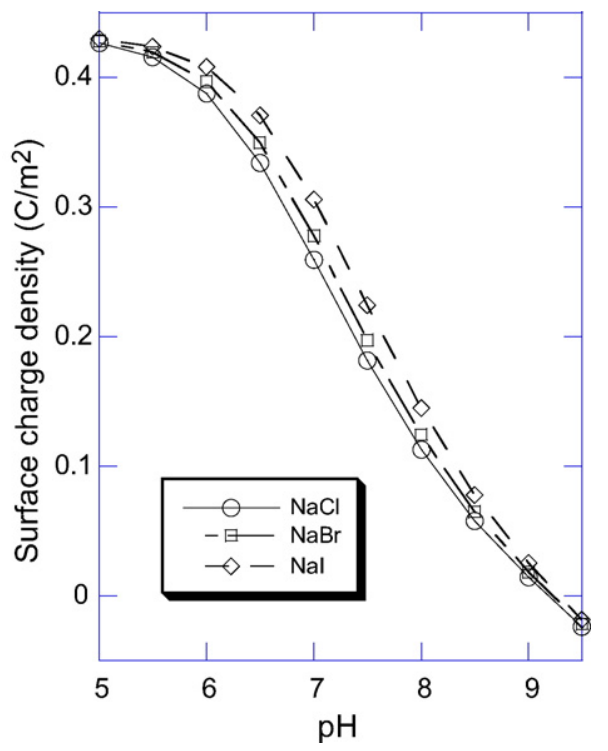

Fig. 4. Surface charge density of an $\mathrm{Al}_{2} \mathrm{O}_{3}$ surface as a function of $\mathrm{pH}$ in 3 different $0.4 \mathrm{M}$ salt solutions. ever, as we will see the net effect from the NES forces on the ion distribution leads to the opposite sequence for $\mathrm{pH}<\mathrm{pI}$.

\section{Extended DLVO theory and forces between oxide surfaces}

The ion distributions between two oxide surfaces can be used to calculate the pressure between two parallel charged membranes. For completeness the expression for the force contribution that originates from the overlapping ion profiles is derived in Appendix A. The double layer pressure between two planar plates a distance $L$ apart can be written as,

$P=k T \sum_{i}\left[c_{i}(L / 2)-c_{o, i}\right]-2 \sum_{i} \int_{x_{o}}^{L / 2} c_{i} \frac{\mathrm{d} U_{i}}{\mathrm{~d} L} \mathrm{~d} x-\frac{H}{6 \pi L^{3}}$,

where $k, T, c_{0, i}, c_{i}(L / 2), x_{0}=2 \AA$, and $U_{i}$ are Boltzmann's constant, temperature, ion density in bulk solution, ion density at the mid-plane between the two surfaces, ion size (which is the closest distance the ions can come to the interface), and the ionic dispersion potential acting between each ion and the two interacting surfaces, respectively. The last term is the direct van der Waals interaction between the two planar surfaces across water. The Hamaker constant $(H)$ values for the interaction between alumina oxide and silica oxide surfaces interacting across water are approximately $5.35 \times 10^{-20} \mathrm{~J}$ and $0.83 \times 10^{-20} \mathrm{~J}$ [44]. The changes in this direct van der Waals term which are due to added salt are included in the two first non-linear terms. It is important to point out that both first two terms in the general case require that fully non-linear modified Poisson-Boltzmann equations, including NES forces, are used to obtain correct results.

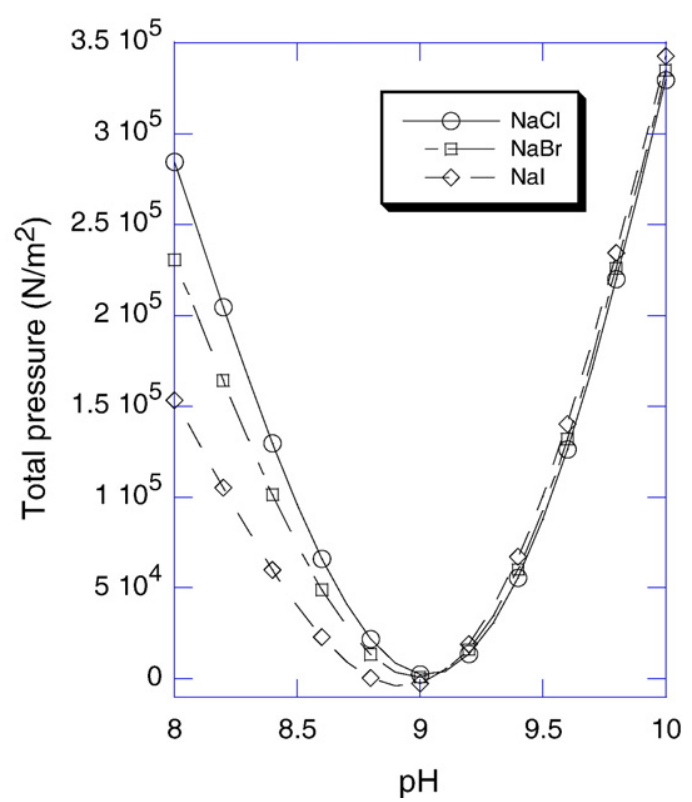

Fig. 5. Total double layer pressure (excluding the direct van der Waals interaction) as a function of $\mathrm{pH}$ between two $\mathrm{Al}_{2} \mathrm{O}_{3}$ surfaces $20 \AA$ apart in different $0.4 \mathrm{M}$ salt solutions. 


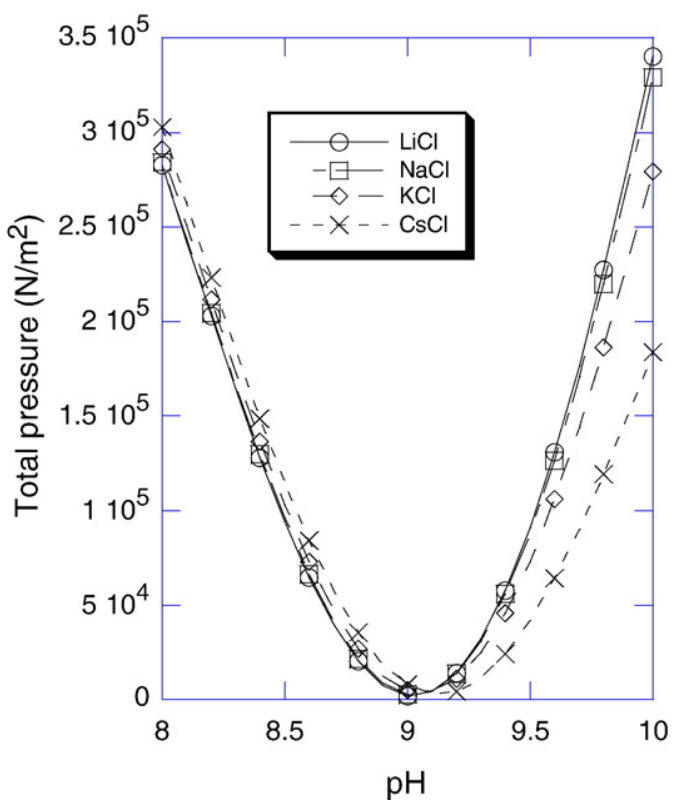

Fig. 6. Total double layer pressure (excluding the direct van der Waals interaction) as a function of $\mathrm{pH}$ between two $\mathrm{Al}_{2} \mathrm{O}_{3}$ surfaces $20 \AA$ apart in different $0.4 \mathrm{M}$ salt solutions.

The ion specific double layer pressures (using Eq. (19) but excluding the direct van der Waals pressure) are shown in Figs. $5-8$ ) as a function of $\mathrm{pH}$ (near the isoelectric points) in various salt solutions for either alumina oxide (anions in Fig. 5 and cations in Fig. 6) or silica oxide surfaces (anions in Fig. 7 and cations in Fig. 8). The theoretical prediction is that the total double layer pressure should become more attractive in the following sequences at $\mathrm{pH}$ below the isoelectric point: $\mathrm{NaCl}<\mathrm{NaBr}<\mathrm{NaI}$ for anions and $\mathrm{CsCl}<\mathrm{KCl}<\mathrm{NaCl}<\mathrm{LiCl}$ for

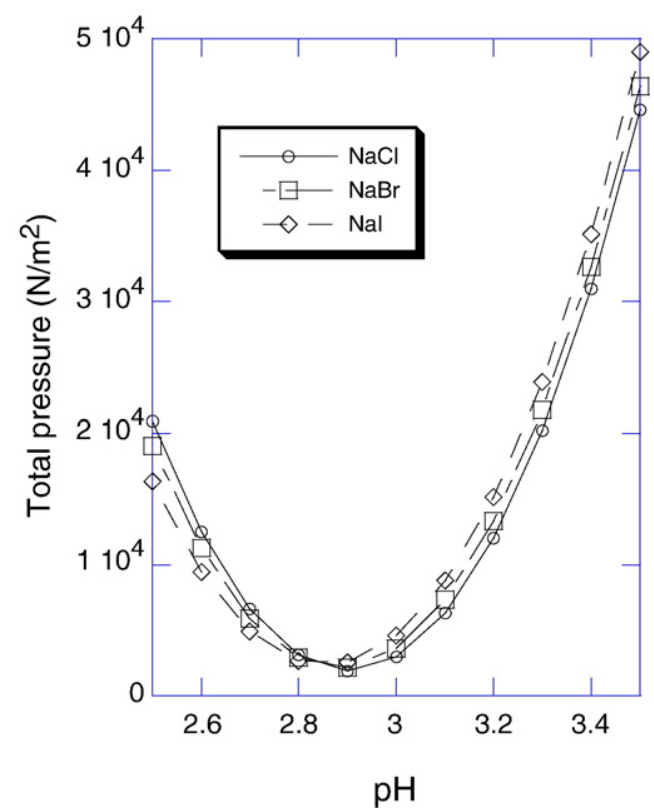

Fig. 7. Total double layer pressure (excluding the direct van der Waals interaction) as a function of $\mathrm{pH}$ between two $\mathrm{SiO}_{2}$ surfaces $20 \AA$ apart in different $0.4 \mathrm{M}$ salt solutions.

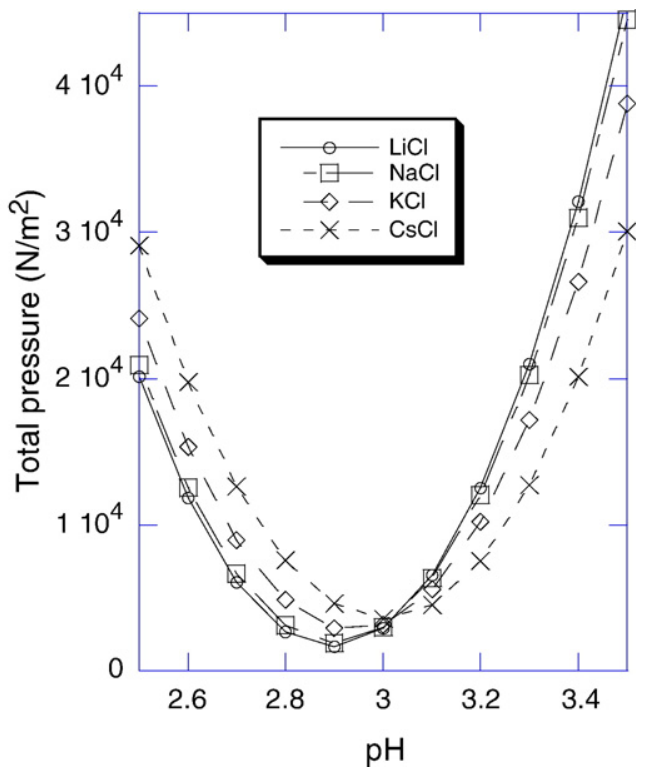

Fig. 8. Total double layer pressure (excluding the direct van der Waals interaction) as a function of $\mathrm{pH}$ between two $\mathrm{SiO}_{2}$ surfaces $20 \AA$ apart in different $0.4 \mathrm{M}$ salt solutions.

cations. Above the isoelectric point the salt ions follow the opposite sequences. These findings are in excellent agreement with the observations made on solubility of various protein solutions $[24-27,61,62]$ where anions follow so-called reversed Hofmeister sequence below the isoelectric point and a direct Hofmeister sequence above the iep.

We show in Fig. 9 the total ion specific double layer pressure at $\mathrm{pH} 8$ between two $\mathrm{SiO}_{2}$ surfaces as a function of surface separation in $0.4 \mathrm{M} \mathrm{LiCl}, \mathrm{NaCl}, \mathrm{KCl}$, and $\mathrm{CsCl}$. The result shows that the interaction becomes more attractive in the

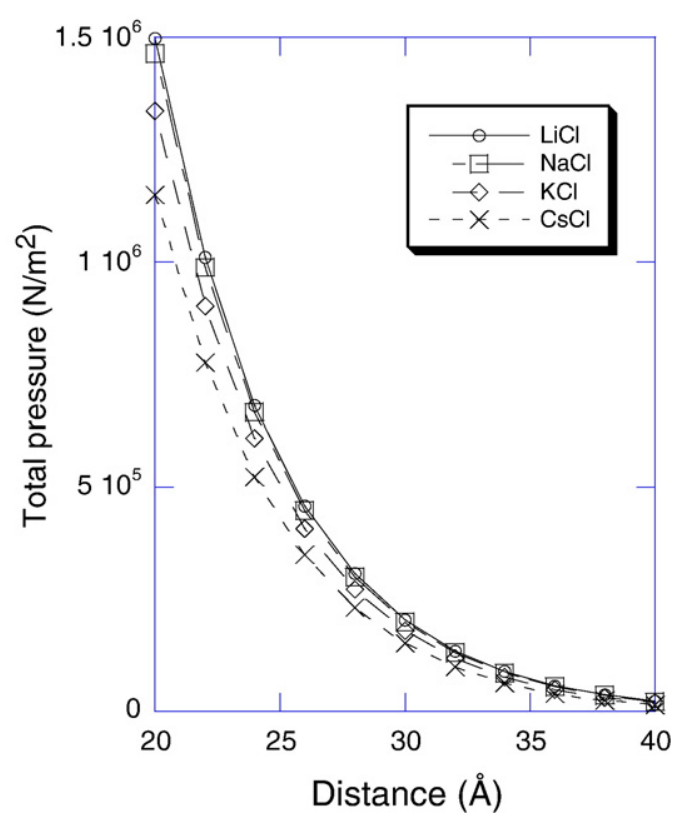

Fig. 9. Total double layer pressure (Eq. (19)) between two $\mathrm{SiO}_{2}$ surfaces (pH 8) as a function of surface separation in different $0.4 \mathrm{M}$ salt solutions. 
sequence $\mathrm{LiCl}<\mathrm{NaCl}<\mathrm{KCl}<\mathrm{CsCl}$ in general agreement with the yield stresses presented in Fig. 6 of [14] (and schematically in our Fig. 1). More polarizable counterions bind more tightly to the surfaces leading to a smaller total concentration of ions in the middle of the two plates. This leads to a larger attraction between the two plates with increasing attractive NES forces acting between counterions and the oxide surface. Our results are also consistent with the zeta potential measurements performed by Franks which become more positive with increasing anion polarizability (Fig. 4) [14]. At low pH, the Hofmeister cation sequence observed in yield stress experiments on silica suspensions is reversed. While the results presented in Figs. 58 show that co-ion effects are quite small, our results do give the right Hofmeister sequence also when the cations are co-ions to the silica oxide surface. Here co-ion adsorption enhances the concentration to such extent that the total number of ions in between the surfaces increases leading to a reduced attraction with increasing attractive NES forces acting on the co-ions. This is a direct consequence of the non-linear coupling between electrostatic and electrodynamic (NES) forces.

The same experimental trends with increasing NES forces acting on the counterions are observed in yield stress experiments on alumina suspensions below the isoelectric point [16]. We show in Fig. 10 that the total double layer pressures between two alumina oxide surfaces at $\mathrm{pH} 6$ in $0.4 \mathrm{M} \mathrm{NaCl}, \mathrm{NaBr}$, and $\mathrm{NaI}$ do give the right Hofmeister sequence compared with experiments. The interaction becomes more attractive as the polarizability of the counterions (and the corresponding NES forces) increases. We have separated the double layer pressure between the same two alumina oxide surfaces ( $\mathrm{pH} 6$ ) across a $0.4 \mathrm{M} \mathrm{NaCl}$ solution into its different contributions in Fig. 11 . One must, in general, treat both non-linear terms in the ion specific double layer pressure in a non-linear theory to obtain correct results. The attractive NES forces acting on the counterions again lead to more attraction between the two surfaces

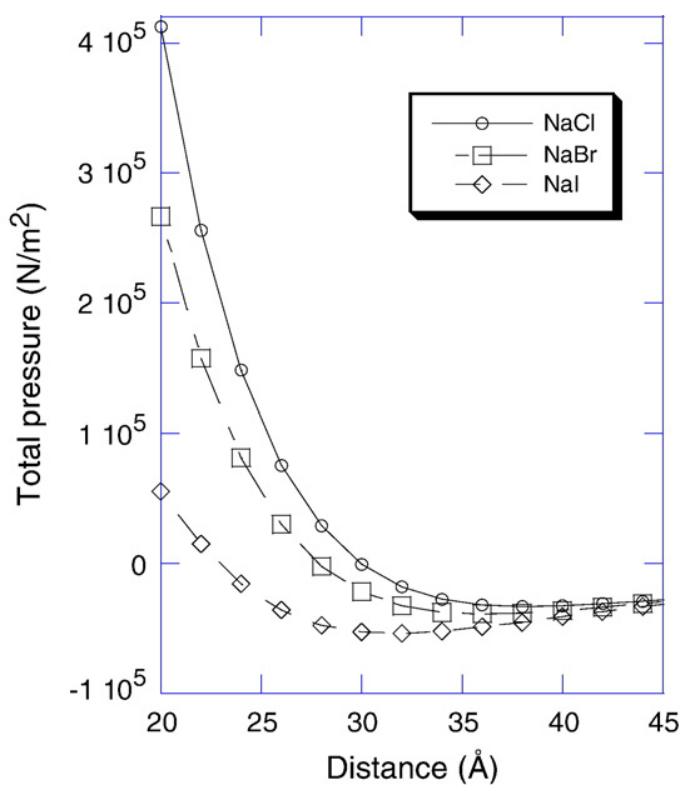

Fig. 10. Total double layer pressure (Eq. (19)) between two $\mathrm{Al}_{2} \mathrm{O}_{3}$ surfaces (pH 6) as a function of surface separation in different $0.4 \mathrm{M}$ salt solutions.

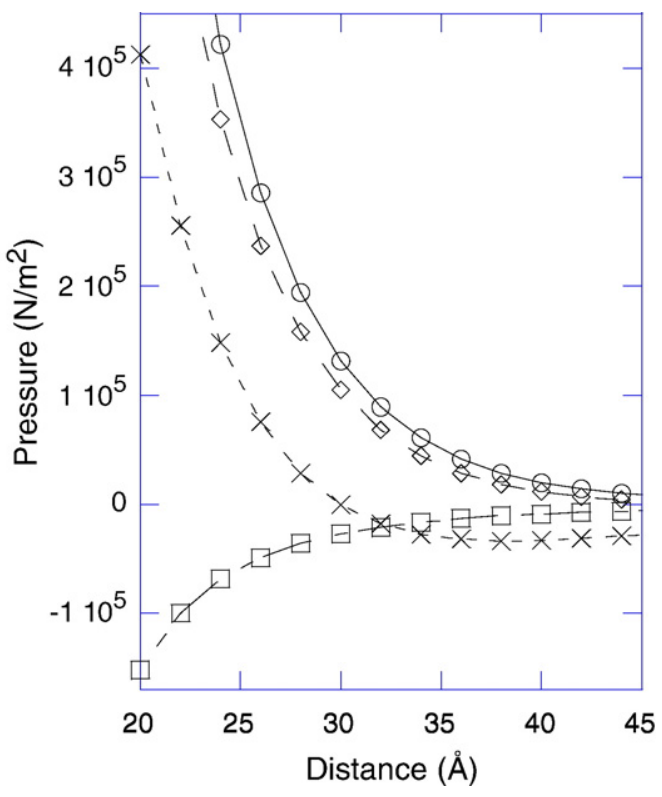

Fig. 11. The different contributions to the total double layer pressure (Eq. (19)) between two $\mathrm{Al}_{2} \mathrm{O}_{3}$ surfaces $(\mathrm{pH} 6)$ as a function of surface separation in a $0.4 \mathrm{M}$ $\mathrm{NaCl}$ solution. The different contributions that we show here are the first nonlinear term in Eq. (19) (circles), the second non-linear term in Eq. (19) (squares), the sum of the two non-linear terms in Eq. (19) (diamonds), and the total double layer pressure (crosses).

and to reduced surface potentials (which we note also explain the experimentally observed zeta potentials in alumina suspensions [16]).

\section{Summary}

We clearly see that inclusion of NES forces provides insights into ion specific Hofmeister effects also in oxide suspensions. In this connection it is interesting to note that condensation of silicate anions in a structural template to form mesoporous silica depends on the background salt solution and on $\mathrm{pH}$ [63]. In the acidic synthesis (low $\mathrm{pH}$ ) the induction time for the precipitation decreases in the order $\mathrm{Cl}^{-}>\mathrm{Br}^{-}>\mathrm{NO}_{3}^{-}$. In alkaline synthesis (high $\mathrm{pH}$ ) the order is roughly the opposite: $\mathrm{ClO}_{3}^{-}>\mathrm{NO}_{3}^{-}>$ $\mathrm{Br}^{-}>\mathrm{Cl}^{-}>\mathrm{F}^{-}$. It is plausible that reconstruction of soft oxide surfaces depends on the choice of background salt solution.

Considering that force measurements and other experiments on different oxide surfaces often give different results under different conditions, we would be very much surprised if the present theoretical results would be able to explain every different rheological experiments. We have, for instance, not taken into account of the gel-like layer that under certain conditions can be formed on the oxide surface. Especially at high $\mathrm{pH}$, hydroxyl groups in solution can enhance the reaction between water with, e.g., silica-oxygen bonds and these reactions depend on the choice of background salt. The bonds may be broken and form silicic acid chains leading to surface gelation that depends, in an intricate way, on the manner of preparation and history [31]. Ions that enter this soft surface region will not only experience simple ionic dispersion potentials as in our approximate formula but one needs in the calculation of the effective NES forces 
which also act on ions to take into account changes in ion selfenergies $[21,22,51,64]$. The surface region itself will, furthermore, be differently influenced by different background salt solutions. At the high particle volume fractions used in the experiments there will also be an influence of ion specific $\mathrm{pH}$ regulation due to the large concentration of surface reactive groups in solution. Ion specific effects on solution pH (M. Boström et al., J. Phys. Chem. B, submitted for publication) and intermolecular forces (M. Boström et al., Biophys. Chem., submitted for publication) have recently been explored in protein solutions by Boström et al. using a Poisson-Boltzmann cell model. We have found that the $\mathrm{pH}$ in protein and buffer solutions depends, in an intricate way, on the choice of background salt (and on the NES forces that act between each ion and protein or buffer present). It is also important to recognize that there, in general, will be both bulk effects (e.g. due to changes in bulk pH due to ionbuffer interactions, as we recently considered, and ion-water interactions [23]) and surface effects discussed here (due to ion-surface interactions).

One example where our simple estimates need to be refined is for the cation effects observed with alumina oxide suspensions at pH's above the iep [15]. Here the experiments as described in our schematic Fig. 1 suggest that the attraction between alumina oxide particles increases in the order $\mathrm{Cs}^{+}<$ $\mathrm{K}^{+}<\mathrm{Na}^{+}<\mathrm{Li}^{+}$. It is clear from Fig. 6 that the theory at the present level of approximation predicts the opposite sequence. So there are still many interesting challenges ahead of us before we fully understand the ion specificity of oxide suspensions. What seems clear is that there, in general, is an important role for NES forces (such as ionic dispersion potentials and ionic solvation energy changes).

\section{Acknowledgements}

M.B. thanks the Swedish Research Council for financial support and F.W. Tavares for stimulating discussions concerning ion specific double layer forces. G.V.F. thanks the Australian Research Council for support (Discovery Grant DP0343326).

\section{Appendix A. Ion specific double layer pressure}

We will for completeness in this appendix derive the expression for the ion specific double layer pressure following closely Burak and Andelman [65] and Edwards and Williams [66]. The free energy of the system is comprised of two parts, the modified Poisson-Boltzmann energy $\left(F_{\mathrm{PB}}\right)$ and the ionic dispersion energy between ions and the two interfaces $\left(F_{\text {disp }}\right)$. Edwards and Williams [66] made the incorrect assumption that one can use a linearized version of Poisson-Boltzmann equation and that the second term should be accounted for in the direct van der Waals interaction (rather than being treated separately in a non-linear theory). This is an unfortunate misunderstanding since correct results clearly require that the fully non-linear theory to be used to obtain the correct ion profiles which is needed for both non-linear energy terms. The essential point is that the electrodynamic ionic dispersion potentials should be treated on the same non-linear level as electrostatic potential.
The modified Poisson-Boltzmann free energy includes both the following terms,

$$
\begin{aligned}
& F_{\mathrm{PB}}=\frac{\varepsilon_{0} \varepsilon_{2}(0)}{2} \int_{\mathrm{a}}^{L-a}\left(\frac{\mathrm{d} \phi}{\mathrm{d} x}\right)^{2} \mathrm{~d} x+k T \int_{\mathrm{a}}^{L-a} \sum_{i} c_{i}(x)\left[\ln \frac{c_{i}(x)}{c_{i, 0}}-1\right] \mathrm{d} x . \\
& F_{\text {disp }}=\int_{\mathrm{a}}^{L-a} \sum_{i} c_{i}(x) U_{i}(x) \mathrm{d} x .
\end{aligned}
$$

Burak and Andelman [65] introduced the trick that one can imagine that the separation between the two plates is increased from a distance $L$ to $L+\delta L$ by inserting a thin slice in the midplane between the plates. The change in free energy (Eq. (A4) of [65] with zero electric field in the midplane) is then,

$$
\begin{aligned}
\delta F_{\mathrm{PB}}= & \delta F_{\mathrm{PB}, 1}+\delta F_{\mathrm{PB}, 2} \\
\delta F_{\mathrm{PB}, 1}= & \delta x\left[\sum_{i}\left\{z_{i} e \phi(L / 2) c_{i}(L / 2)+k T c_{i}(L / 2)\left[\ln \frac{c_{i}(L / 2)}{c_{i, 0}}-1\right]\right\}\right] \\
\delta F_{\mathrm{PB}, 2}= & \int_{\mathrm{a}}^{L-a} \mathrm{~d} x \sum_{i} \delta c_{i}\left[z_{i} e \phi(x)+k_{\mathrm{B}} T \ln \frac{c_{i}(x)}{c_{i, 0}}\right] \\
\delta F_{\mathrm{disp}}= & \delta x \sum_{i} c_{i}(L / 2) U_{i}(L / 2)+\int_{\mathrm{a}}^{L-a} \mathrm{~d} x \sum_{i} \delta c_{i} U_{i}(x) \\
& +2 \delta x \int_{\mathrm{a}}^{L / 2} \mathrm{~d} x \sum_{i} c_{i} \frac{\mathrm{d} U_{i}(x)}{\mathrm{d} L} .
\end{aligned}
$$

These expressions can be simplified using the Boltzmann equation in the forms [66],

$$
\begin{aligned}
& k_{\mathrm{B}} T \ln \left\lfloor c_{i}(x) / c_{i, 0}\right\rfloor=-z_{i} e \phi(x)-U_{i}(x) \\
& \delta F_{\mathrm{PB}, 1}=\delta x\left[\sum_{i}\left\{c_{i}(L / 2)\left[-U_{i}(L / 2)\right]-k T c_{i}(L / 2)\right\}\right],
\end{aligned}
$$

$\delta F_{\mathrm{PB}, 2}=\int_{\mathrm{a}}^{L-a} \mathrm{~d} x \sum_{i} \delta c_{i}\left[-U_{i}(x)\right]$.

The total double layer pressure due to the non-linear ion profile can then be written as

$$
\begin{aligned}
P & =-\delta\left(F_{\text {disp }}+F_{\mathrm{PB}}\right) / \delta x \\
& =\sum_{i} k T c_{i}(L / 2)-\int_{\mathrm{a}}^{L / 2} \mathrm{~d} x \sum_{i} 2 c_{i} \frac{\mathrm{d} U_{i}(x)}{\mathrm{d} L} .
\end{aligned}
$$

To obtain the net pressure on the plates given as Eq. (19) one should finally subtract the pressure on the outside of the plates which is $\sum_{i} k T c_{i, 0}$. The first term in Eq. (A7) looks identical to the classical textbook expression [67] in which the osmotic pressure between two equal plates is given by the ion density in the midplane. What is new is that there are now two terms and 
both depend in a very ion specific way on the non-linear ion profiles. One should note that this derivation did not include the specific nature of the non-electrostatic (or electrostatic) forces that contribute to $U_{i}(x)$. This means that one can, for example, directly extend the present calculation to include image potentials by simply adding a term with the derivative of the image potential [47] acting on the ions between two planes and, of course, then also add the image potential in the modified nonlinear Poisson-Boltzmann equation.

We will finally in this appendix derive contributions to the second pressure term from both ionic dispersion potentials and image potentials acting on ions in solution. We approximate the ionic dispersion potential acting on an ion between two plates with,

$U_{\text {dispersion }}(x)=\frac{B}{x^{3}}+\frac{B}{(L-x)^{3}}$,

this means that the derivative in Eq. (A8) gives,

$\frac{\mathrm{d} U_{\text {dispersion }}(x)}{\mathrm{d} L}=\frac{-3 B}{(L-x)^{4}}$.

The image potentials [47] acting on an ion in solution between the two plates can be written as,

$U_{\text {image }}(x)=\frac{e^{2}\left(S_{i}+S_{2}\right)}{16 \pi \varepsilon_{0} \varepsilon_{2}(0)}$,

$S_{1}=-2 \ln \left[1-\Delta_{12} \exp (-2 \kappa L)\right] / L$,

$S_{2}=\Delta_{12}^{-1} \sum_{p=1}^{\infty}\left[\frac{e^{-2 \kappa[L(p-1)+x]}}{L\left(p^{-1}\right)+x}+\frac{e^{-2 \kappa[L p-x]}}{L p^{-x}}\right]$,

where $\kappa$ is the inverse Debye-length. This means that the contribution to the non-linear pressure from image potentials is only slightly more complicated than the part from ionic dispersion potentials. The derivatives of $S_{1}$ and $S_{2}$ are,

$\frac{\mathrm{d} S_{1}}{\mathrm{~d} L}=\frac{2 \Delta_{12} \kappa e^{-2 \kappa L}}{\left(1-\Delta_{12} \kappa e^{-2 \kappa L}\right) L}-\ln \left[1-\Delta_{12} \exp (-2 \kappa L)\right] / L^{2}$

$$
\begin{aligned}
\frac{\mathrm{d} S_{2}}{\mathrm{~d} L}= & \sum_{p=1}^{\infty}\left[\frac{e^{-2 \kappa[L(p-1)+x]}(p-1)\{1+2 \kappa[L(p-1)+x]\}}{[L(p-1)+x]^{2} \Delta_{12}}+\right] \\
& +\sum_{p=1}^{\infty}\left[\frac{e^{-2 \kappa[L p-x]}\left\{1-2 \kappa L p^{2}+p[2 \kappa(L-x)-1]\right\}}{[L(p-1)+x]^{2} \Delta_{12}}\right]
\end{aligned}
$$

It is important to be consistent, i.e. a calculation that includes image potential in the Poisson-Boltzmann equation should also include contributions to the pressure from

$$
\frac{\mathrm{d} U_{\text {image }}(x)}{\mathrm{d} L}=\frac{e^{2}\left(\mathrm{~d} S_{1} / \mathrm{d} L+\mathrm{d} S_{2} / \mathrm{d} L\right)}{16 \pi \varepsilon_{0} \varepsilon_{2}(0)} .
$$

In general the second term in Eq. (A8) should include one contribution from each additional potential that one takes into account in the extended Poisson-Boltzmann equation. In Section 7 we take into account the ionic dispersion potential that acts between each ion in solution and the two oxide surfaces.

\section{References}

[1] Kunz W, Lo Nostro P, Ninham BW. Curr Opin Colloid Interface Sci 2004;9:1.

[2] Israelachvili JN, Adams GE. Nature 1976;262:77.

[3] Pashley RM. J Colloid Interface Sci 1981;83:531.

[4] Pashley RM, McGuiggan PM, Ninham BW, Brady J, Evans DF. J Phys Chem 1986;90:1637.

[5] Shubin VE, Kékicheff P. J Colloid Interface Sci 1993;155:108.

[6] Horn RG, Smith DT. J Non-Cryst Solids 1990;120:72.

[7] Chapel JP. Langmuir 1994;10:4237.

[8] Vakarelski IU, Ishimura K, Higashitani K. J Colloid Interface Sci 2000;227:111.

[9] Vakarelski IU, Higashitani K. J Colloid Interface Sci 2001;242:110.

[10] Donose BC, Vakarelski IU, Higashitani K. Langmuir 2005;21:1834.

[11] Craig VSJ, Ninham BW, Pashley RM. Nature 1993;364:317.

[12] Vogel R. Curr Opin Colloid Interface Sci 2004;9:133.

[13] Lo Nostro P, Ninham BW, Lo Nostro A, Pesavento G, Fratoni L, Baglioni P. Phys Biol 2005;2:1.

[14] Franks GV. J Colloid Interface Sci 2002;249:44.

[15] Johnson SB, Franks GV, Scales PJ, Healy TW. Langmuir 1999;15:2844.

[16] Franks GV, Johnson SB, Scales PJ, Boger DV, Healy TW. Langmuir 1999;15:4411.

[17] Franks GV, Meagher L. Colloids Surf, A Physicochem Eng Asp 2003;214:99.

[18] Kim H-K, Tuite E, Nordén B, Ninham BW. Eur Phys J, E Soft Matter 2001;4:411.

[19] Boström M, Williams DRM, Ninham BW. Biophys J 2003;85:686.

[20] Weissenborn PK, Pugh RJ. J Colloid Interface Sci 1996;184:550.

[21] Boström M, Williams DRM, Ninham BW. Langmuir 2001;17:4475.

[22] Boström M, Kunz W, Ninham BW. Langmuir 2005;21:2619.

[23] Jungwirth P, Tobias D. J Phys Chem B 2001;105:10468.

[24] Riés-Kautt MM, Ducruix AF. J Biol Chem 1989;264:745.

[25] Carbonnaux C, Riés-Kautt MM, Ducruix A. Protein Sci 1995;5:2123.

[26] Finet S, Skouri-Panet F, Casselyn M, Bonneté F, Tardieu A. Curr Opin Colloid Interface Sci 2004;9:112.

[27] Bonneté F, Finet S, Tardieu A. J Cryst Growth 1999;196:403.

[28] Collins KD, Washabaugh MW. Q Rev Biophys 1985;18:323.

[29] Collins KD. Methods 2004;34:300.

[30] Vigil G, Xu ZH, Steinburg S, Israelachvili JN. J Colloid Interface Sci 1994;165:367.

[31] Yaminsky VV, Ninham BW. Langmuir 1998;14:3223.

[32] Adler JJ, Rabinovich YI, Moudgil BM. J Colloid Interface Sci 2001;237:249.

[33] Li Y, Kanda Y, Shinto H, Vakarelski IU, Highshitani K. Colloids Surf, A Physicochem Eng Asp 2005;260:39.

[34] Mahanty J, Ninham BW. Dispersion forces. London: Academic Press; 1976.

[35] Ninham BW, Yaminsky V. Langmuir 1997;13:2097.

[36] Boström M, Williams D, Ninham BW. Phys Rev Lett 2001;87:168103.

[37] Tavares FW, Bratko D, Blanch HW, Prausnitz JM. J Phys Chem B 2004;208:9228.

[38] Ninham BW, Parsegian VA. J Theor Biol 1971;31:495.

[39] Johnson SB, Franks GV, Scales PJ, Boger DV, Healy TW. Int J Miner Process 2000;58:267.

[40] Tadros ThF, Lyklema J. J Electroanal Chem 1968;17:267.

[41] Sprycha R. J Colloid Interface Sci 1989;127:1.

[42] Nir S. Prog Surf Sci 1976;8:1.

[43] Grabbe A. Langmuir 1993;9:797.

[44] Bergström L, Meurk A, Arwin H, Rowcliffe DJ. J Am Ceram Soc 1996;79:339.

[45] Parsegian VA, Ninham BW. Biophys J 1970;10:664.

[46] Boström M, Sernelius Bo E. Physica, A 2004;339:53.

[47] Boström M, Sernelius Bo E. Phys Rev Lett 2000;84:4757.

[48] Ninham BW, Boström M. Phys Rev A 2003;67:030701. 
[49] Boström M, Longdell JJ, Mitchell DJ, Ninham BW. Eur Phys J, D At Mol Opt Phys 2003;22:47.

[50] Boström M, Ninham BW. J Phys Chem B 2004;108:12593.

[51] Boström M, Ninham BW. Biophys Chem 2005;114:95.

[52] Karraker KA, Radke CJ. Adv Colloid Interface Sci 2002;96:231.

[53] James RO. Adv Ceram 1987;21:349.

[54] Boström M, Williams D, Ninham BW. Langmuir 2002;18:8609.

[55] Boström M, Williams D, Stewart PR, Ninham BW. Phys Rev E 2003;68:041902.

[56] Boström M, Williams D, Ninham BW. Europhys Lett 2003;63:610.

[57] Boström M, Williams D, Ninham BW. Langmuir 2002;18:6010.

[58] Boström M, Williams D, Ninham BW. J Phys Chem B 2002;106:7908.

[59] Lo Nostro P, Fratoni LU, Ninham BW, Baglioni P. Biomacromolecules 2002;3:1217.
[60] Lo Nostro P, Lo Nostro A, Ninham BW, Pesavento G, Fratoni L, Baglioni P. Curr Opin Colloid Interface Sci 2004;9:97.

[61] Gustavson HK. In: Boyer H, editor. Colloidal symposium monograph. New York: The Chemical Catalog Company Inc.; 1926.

[62] Loeb J. Science 1920;LII:449.

[63] Lin H-P, Kao C-P, Mou C-Y. Microporous Mesoporous Mater 2001;48:135.

[64] Boström M, Ninham BW. Langmuir 2004;20:7569.

[65] Burak Y, Andelman D. J Chem Phys 2001;114:3271.

[66] Edwards S, Williams D. Phys Rev Lett 2004;92:248303-1.

[67] Evans DF, Wennerström H. The Colloidal Domain. Where physics, chemistry, biology, and technology meet. New York: Wiley-VCH; 1999. 